\title{
Rapid, reversible activation of AgRP neurons drives feeding behavior in mice
}

\author{
Michael J. Krashes, ${ }^{1}$ Shuichi Koda, ${ }^{1,2}$ ChianPing Ye, ${ }^{1}$ Sarah C. Rogan, ${ }^{3}$ Andrew C. Adams, ${ }^{1}$ \\ Daniel S. Cusher, ${ }^{1}$ Eleftheria Maratos-Flier, ${ }^{1}$ Bryan L. Roth, ${ }^{3}$ and Bradford B. Lowell ${ }^{1}$ \\ ${ }_{1}^{1}$ Division of Endocrinology, Department of Medicine, Beth Israel Deaconess Medical Center, Harvard Medical School, Boston, Massachusetts, USA. \\ ${ }^{2}$ Asubio Pharma, Kobe, Japan. ${ }^{3}$ Department of Pharmacology, Psychiatry and Medicinal Chemistry and the Program in Neurosciences, \\ University of North Carolina School of Medicine, Chapel Hill, North Carolina, USA.
}

\begin{abstract}
Several different neuronal populations are involved in regulating energy homeostasis. Among these, agoutirelated protein (AgRP) neurons are thought to promote feeding and weight gain; however, the evidence supporting this view is incomplete. Using designer receptors exclusively activated by designer drugs (DREADD) technology to provide specific and reversible regulation of neuronal activity in mice, we have demonstrated that acute activation of AgRP neurons rapidly and dramatically induces feeding, reduces energy expenditure, and ultimately increases fat stores. All these effects returned to baseline after stimulation was withdrawn. In contrast, inhibiting AgRP neuronal activity in hungry mice reduced food intake. Together, these findings demonstrate that AgRP neuron activity is both necessary and sufficient for feeding. Of interest, activating AgRP neurons potently increased motivation for feeding and also drove intense food-seeking behavior, demonstrating that AgRP neurons engage brain sites controlling multiple levels of feeding behavior. Due to its ease of use and suitability for both acute and chronic regulation, DREADD technology is ideally suited for investigating the neural circuits hypothesized to regulate energy balance.
\end{abstract}

\section{Introduction}

Obesity is an epidemic that continues to grow unabated in developed nations where an abundance of calorically dense foods are plentiful and easily obtained. This excess of food is a major contributor to high levels of obesity, which is a major risk factor for cardiovascular disease, certain types of cancer, and type 2 diabetes. Through neuroscience research, critical contributions can be made in developing an understanding of the mechanisms underlying this problem, ultimately leading to treatment of this escalating disorder (1).

Agouti-related protein (AgRP) neurons are located in the arcuate nucleus (ARC) of the hypothalamus and have been implicated in regulating energy homeostasis (2). They release AgRP, neuropeptide Y (NPY) and $\gamma$-aminobutyric acid (GABA), all of which have been linked with changes in energy balance (2-5). Despite evidence for their involvement in feeding behavior and energy balance, the exact contribution of these neurons remains unclear, since chronic and acute perturbations of AgRP neurons and the factors they release have produced inconsistent results. Overexpression of AgRP in transgenic mice produces obesity, and central administration of the orexigenic peptides, AgRP or NPY, causes hyperphagia $(2,3)$. However, targeted deletion of the genes encoding AgRP, NPY, and/or vesicular GABA transporter (VGAT; required for release of GABA) has minimal effects on feeding (4-6). Likewise, acute ablation of AgRP neurons in neonates or delayed degeneration of AgRP neurons during adulthood has little impact on energy metabolism (7-9). Conversely, acute ablation of AgRP neurons in adult mice, a manipulation associated with gliosis $(5)$, induces severe hypophagia $(7,8)$. Given the above,

Authorship note: Michael J. Krashes and Shuichi Koda contributed equally to this work.

Conflict of interest: The authors have declared that no conflict of interest exists. Citation for this article: J Clin Invest. 2011;121(4):1424-1428. doi:10.1172/JCI46229. manipulating AgRP neurons in a manner that is selective, rapid, and reversible, should provide insight, both qualitative and quantitative, into the function of these neurons.

\section{Results and Discussion}

One way to accomplish this is via designer receptors exclusively activated by designer drugs (DREADD) technology, in which muscarinic GPCRs are mutated so that their ability to bind natural ligands is lost, while nanomolar potency is gained for the otherwise pharmacologically inert ligand clozapine-N-oxide (CNO) $(10,11)$. The stimulatory DREADD, designated "hM3Dq", couples through the Gq pathway to depolarize neurons $(10,11)$. Here, we used a Cre-recombinase-dependent adeno-associated virus (AAV) to target hM3Dq to AgRP neurons (Figure $1 \mathrm{~A})$. The DREADD was fused to mCherry so that receptor expression could be monitored. Stable transgene inversion was achieved using the FLEX Switch (12). When the AAV-hM3Dq-mCherry was stereotaxically injected into the ARC of AgRP-Ires-cre mice, mCherry was detected exclusively in the ARC, in a pattern consistent with AgRP neurons (Figure 1B). Importantly, no expression was detected following injection into non-Cre-expressing mice (data not shown). Furthermore, mCherry was restricted to AgRP-Cre-expressing cells, as revealed by colocalization with GFP in mice also harboring a Cre-dependent GFP reporter transgene (Figure 1C) (>95\% of GFP-expressing neurons express mCherry). Notably, GFP protein is cytoplasmic, while the DREADD receptor is expressed on the plasma membrane. Whole cell, current clamp recordings were then performed in mCherry-expressing AgRP neurons. When added to the brain slice, $\mathrm{CNO}$ depolarized and markedly increased the firing rate of AgRP neurons (Figure 1D). The effect of CNO-induced depolarization was reversible following washout (Figure 1D). Importantly, CNO did not affect the membrane potential of nonDREADD-expressing AgRP neurons (data not shown). When injected in vivo, CNO greatly increased c-fos immunoreactivity 
A

\section{L-ITR human synapsin}

\section{Кมวบวแ-baรพบ}

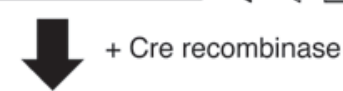

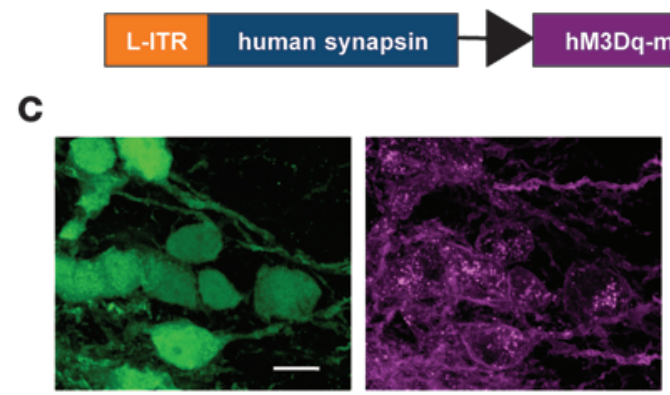

D

CNO $(5 \mu \mathrm{M})$

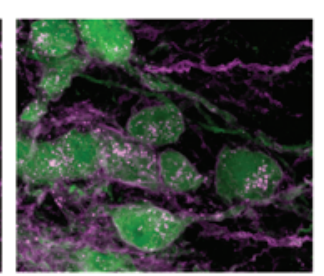

.

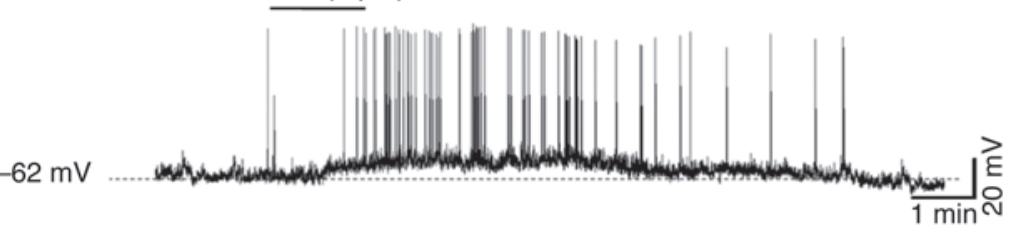

E

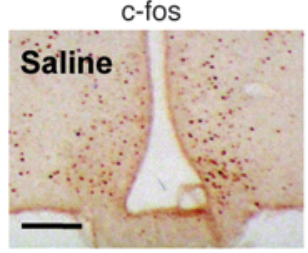

B

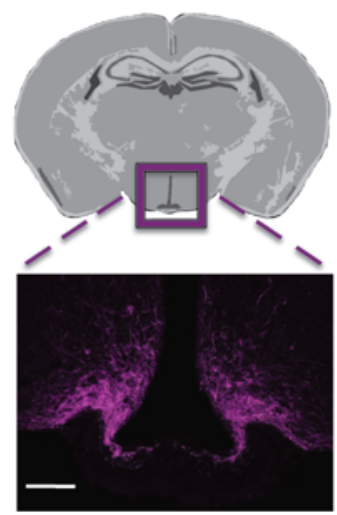

Figure 1

Cre-dependent AAV-hM3Dq-mCherry is specifically expressed in the ARC of AgRP-Ires-cre mice and confers activation by CNO. (A) Design of hM3Dq-mCherry AAV employing the FLEX Switch strategy, which uses 2 pairs of heterotypic, antiparallel loxP-type recombination sites to achieve Cre-mediated transgene inversion and expression (12). L-ITR, left-inverted terminal repeat; R-ITR, right-inverted terminal repeat; WPRE, woodchuck hepatitis posttranscriptional regulatory element. (B) Top: Schematic indicating the site of the imaged area in the ARC of the hypothalamus. Bottom: mCherry fluorescence exclusively in the ARC after bilateral injections of AAV-hM3Dq-mCherry into the hypothalamus of AgRP-Ires-cre mice crossed with Z/EG Cre-dependent reporter mice. Z/EG mice expressed GFP protein following Cre-mediated excision of an intervening sequence (Scale bar: $100 \mu \mathrm{m}$ ). (C) Colocalization of mCherry (anti-dsRed) and anti-GFP fluorescence in the ARC. Note that GFP is cytoplasmic and DREADD is expressed on the plasma membrane (scale bar: $10 \mu \mathrm{m}$ ). (D) Whole cell, current clamp recording from an AgRP neuron marked by mCherry fluorescence from an AgRP-Ires-cre mouse injected with AAV-hM3Dq-mCherry. CNO (5 $\mu \mathrm{M})$ elicited rapid depolarization of the membrane potential and greatly increased the firing rate. This example trace is representative of 4 similar recordings. (E) Injection of CNO in vivo induces c-fos immunoreactivity in the ARC. Brains were obtained for c-fos analysis 90 minutes following injection of saline or CNO $(0.3 \mathrm{mg} / \mathrm{kg}$ of body weight, i.p.) (scale bars: $120 \mu \mathrm{m})$.

in the ARC in a pattern consistent with AgRP-expressing neurons (Figure 1E), further evidence that this approach acutely and specifically stimulates neuronal activity.

One critical advantage of this approach, as it applies to investigating in vivo behavior, is that the same animal can be used as its own control (e.g., saline versus $\mathrm{CNO}$ ). As an additional control, we also injected wild-type littermate animals with AAV-hM3DqmCherry virus into the ARC. Thus, all mice shown in Figures 2 and 3 had received bilateral injections of AAV-DREADD-mCherry. However, only the AgRP-Ires-cre mice expressed DREADD in AgRP neurons. Stimulation of AgRP neurons via i.p. injection of $\mathrm{CNO}(0.3 \mathrm{mg} / \mathrm{kg})$ rapidly (in a matter of minutes) induced feeding (see Supplemental Video 1; supplemental material available online with this article; doi:10.1172/JCI46229DS1). Mice fed ad lib were injected during the light phase, a time in which mice normally refrain from eating. CNO-injected AgRP-Ires-cre mice ate nearly 4 -fold more than controls in the first $30 \mathrm{~min}$ utes after injection and continued to eat at a higher rate for the duration of the study (Figure 2A). Furthermore, stimulation of AgRP neurons produced a sharp decline in energy expenditure (Figure 2B), which lasted for approximately 8 hours. Notably, this duration of action for a single injection of CNO is consistent with a previous report (11).
Chronic stimulation of AgRP neurons via twice-daily injections of CNO caused marked weight gain (Figure 2C). AgRPIres-cre mice injected with $\mathrm{CNO}$ gained $2.5 \mathrm{~g}$ following 1 day of treatment and continued to amass weight at a reduced rate until $\mathrm{CNO}$ injections were stopped on day 11 (after 5 days of $\mathrm{CNO}$ ), at which point the AgRP-Ires-cre mice had gained $4.6 \mathrm{~g}$ of body weight (Figure 2C), including $2.9 \mathrm{~g}$ of fat (Figure 2D). This substantial weight gain was due to increased food intake (Figure 2E) and, most likely, also decreased energy expenditure (Figure 2B). Of interest, the CNO-induced obesity was completely reversed following 5 days of drug withdrawal (Figure 2, C and D), and this reversal was associated with hypophagia (Figure $2 \mathrm{E}$ ). Complete normalization of body weight and adiposity, caused in part by "compensatory" hypophagia, strongly supports the existence of a set point for these parameters (13).

The above studies strongly support the sufficiency of AgRP neurons to promote body weight gain via increases in food intake and decreases in energy expenditure. To further address whether these same neurons are required for feeding behavior, we used an identical viral approach in which we subsequently expressed the Gi/o-coupled inhibitory DREADD, designated "hM4Di", in AgRP neurons $(10,11,14)$. As assessed by whole cell, current clamp recordings, $\mathrm{CNO}$ both hyperpolarized and decreased the firing 
A
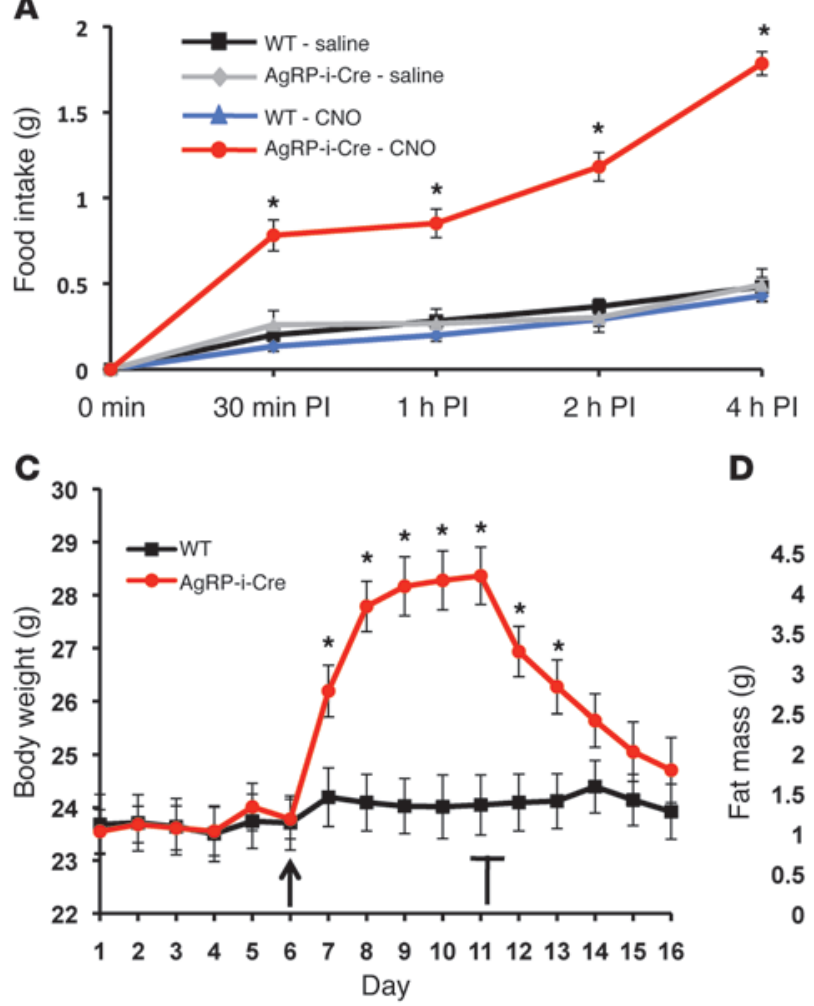

F

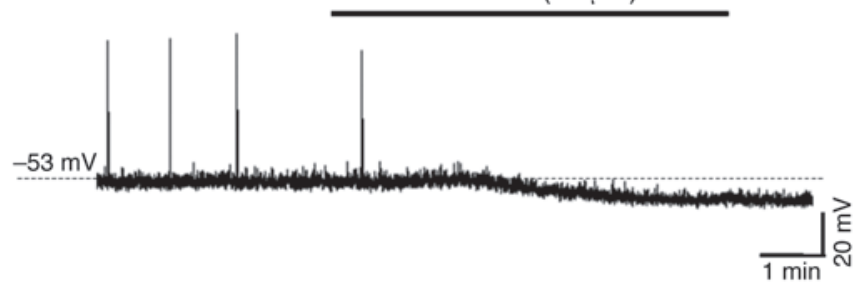

D
B

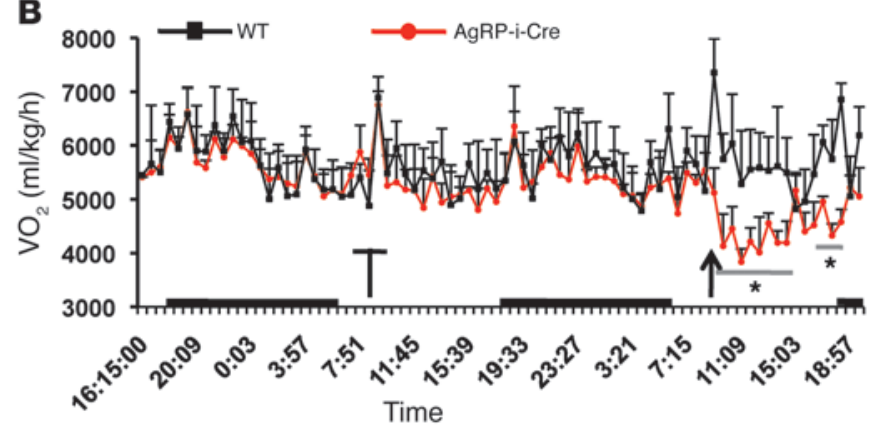

E
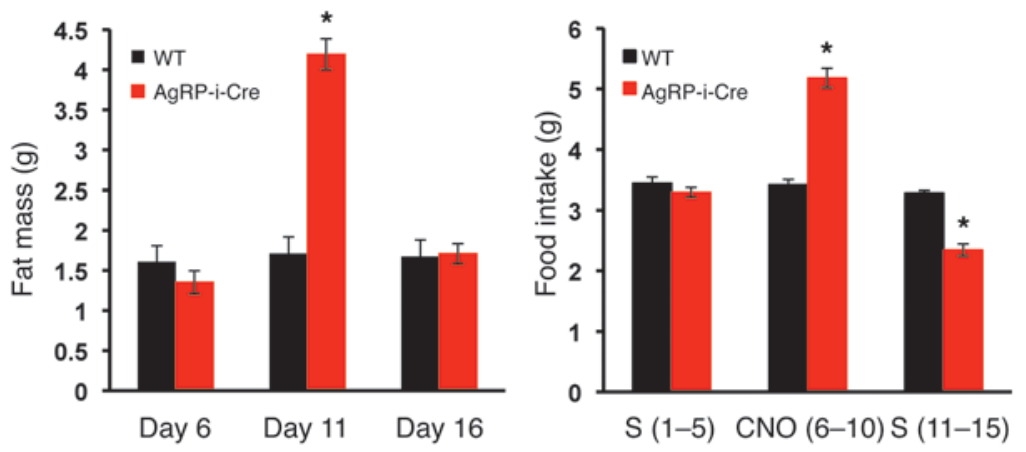

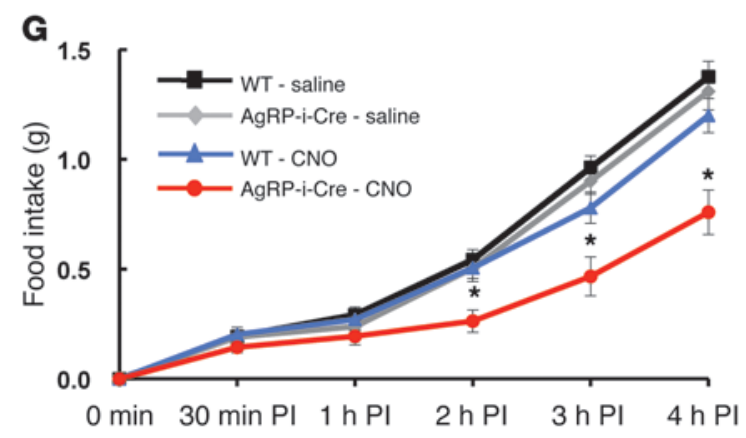

Figure 2

Manipulation of AgRP neuron activity alters energy balance. (A) Food intake. CNO (0.3 mg/kg of body weight, i.p.) or saline was injected 3 hours after the start of the 12-hour light cycle, and food intake was assessed between 30 minutes and 4 hours after injection (PI). Data are from male mice (mean \pm SEM, $n=12$; ${ }^{*} P<0.01$ ). AgRP-i-Cre, AgRP-Ires-cre mice. (B) Oxygen consumption. Mice were acclimated in metabolic cages and injected with either saline (blunted arrow) or CNO (arrow) at 8:30 am. Black bars along the $x$ axis indicate the 12-hour dark cycle. Data are from male mice (mean \pm SEM, $n=6$; ${ }^{*} P<0.01$ ). (C-E) Chronic stimulation of AgRP neurons. (C) Body weight, (D) fat mass, and (E) food intake. AgRP-Ires-cre and wild-type control mice were injected twice daily (at 9:00 am and 5:00 pm) with saline from days 1-5, CNO (0.3 mg/kg of body weight, i.p.) from days 6-10 (arrow), and saline from days 11-15 (blunted arrow). Data are from female mice (mean \pm SEM, $n=12 ;{ }^{*} P<0.01$ ). ( $F$ and $\mathbf{G}$ ) Inhibitory DREADD (hM4Di). (F) Whole cell, current clamp recording from an AgRP neuron marked by mCherry fluorescence from a AgRP-Ires-cre mouse injected with AAV-hM4Di-mCherry. CNO $(10 \mu \mathrm{M})$ hyperpolarized the membrane potential and decreased the firing rate. This example trace is representative of 5 similar recordings. $(G)$ Inhibition of AgRP neurons decreases food intake. CNO (0.3 mg/kg of body weight, i.p.) or saline was injected at the start of the 12-hour dark cycle, and food intake was assessed between 30 minutes and 4 hours PI. Data are from male mice (mean $\pm \mathrm{SEM}, n=6 ;{ }^{\star} P<0.05$ ).

rate of hM4Di-expressing AgRP neurons (Figure 2F). Notably, inhibition of AgRP neurons in vivo at the onset of the dark cycle (when the majority of food intake normally occurs) significantly reduced food intake (Figure $2 \mathrm{G}$ ).

It has been suggested that the hypothalamic neurocircuitry responsible for integrating metabolic signals is embedded within a complex network that permits both the adaptation and synchronization of nutrient needs to conditions in the animal's local environment (15). It is likely that these same neurons invoke neural mechanisms of reward, motivation, and decision making, all of which are integrated to serve the ultimate goal of obtaining and ingesting food. To determine whether these more complex aspects of feeding behavior are engaged by basal hypothalamic neurons, we stimulated AgRP neurons in mice fed ad lib and then assessed their motivation to work (e.g., via a nose poke beam-break setup) for food reinforcements using a progressive ratio operant condi- 
A

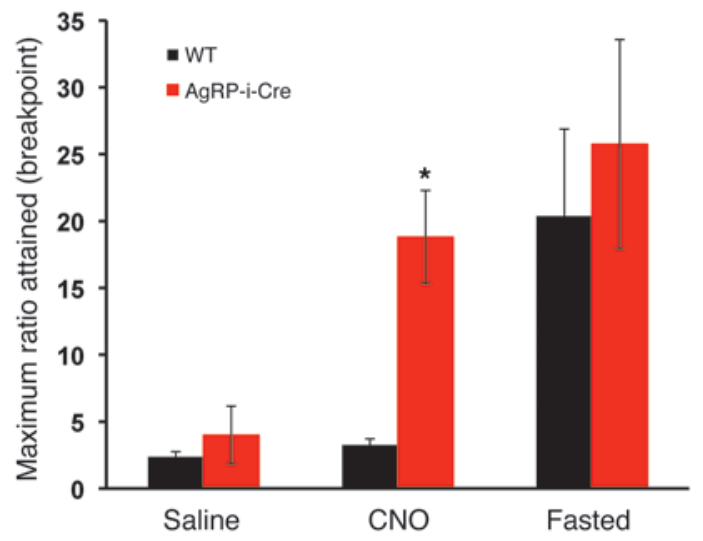

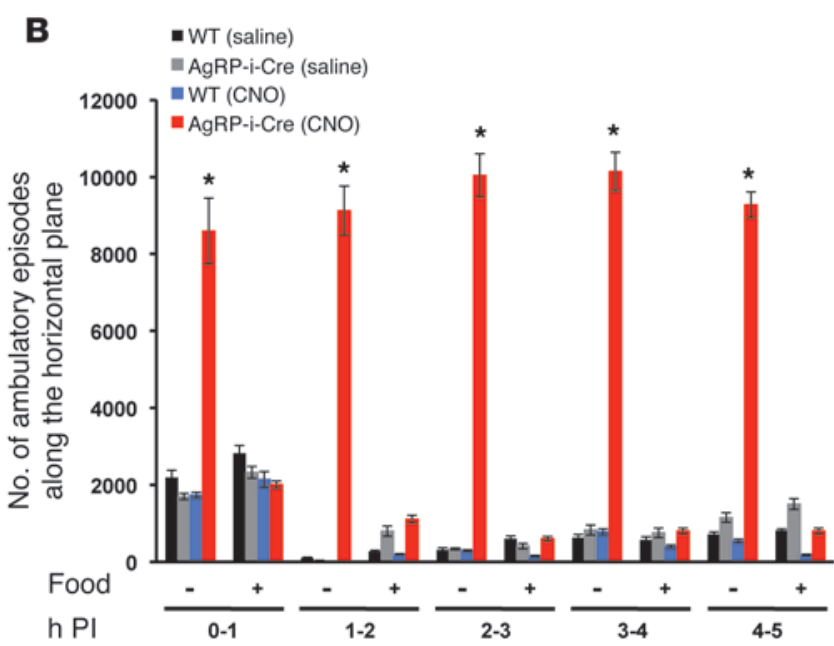

Figure 3

Stimulating AgRP neurons drives a behavioral program to work for and search for food. (A) Ad lib-fed animals injected with saline were first trained to associate a successful nose poke with a reward pellet using an FR1 schedule. Following the training period, the same cohort was injected with CNO $(0.3 \mathrm{mg} / \mathrm{kg}$ of body weight, i.p.) or saline and then tested on a PR3. Acute stimulation of AgRP neurons in mice fed ad lib led to a significant increase in the break point, similar to the break point observed in fasted mice. Data shown are from male mice (mean $\pm \mathrm{SEM}, n=6$, $\left.{ }^{\star} P<0.01\right)$. (B) Stimulation of AgRP neurons increases physical activity when food is absent but not when food is present. In the food-absent study, food was removed immediately following CNO or saline injection. The number of ambulatory episodes along the horizontal plane was assessed 0-1 hour, 1-2 hours, 2-3 hours, 3-4 hours, and 4-5 hours PI. The same cohort of mice was used in the food - and food + studies. Data shown are from male mice (mean \pm SEM, $n=4,{ }^{*} P<0.01$ ).

tioning paradigm (16). In this assay, an animal's willingness to work for food is represented by its breakpoint, the highest number of successive nose pokes a mouse will perform, in a progressively increasing paradigm (i.e., $1,4,7$ ), to procure a single food pellet. For these operant conditioning studies, all mice were trained in the absence of CNO on a fixed ratio of 1 (FR1: 1 nose poke/1 pellet) for 5 consecutive days to learn to correctly associate a nose poke with the delivery of food reinforcement. The motivation to obtain food reinforcement was then assessed using a progressive ratio of 3 (PR3). When AgRP neurons were activated by CNO, mice reached a dramatically higher breakpoint (Figure 3A), which was similar in magnitude to that induced by the calorically deficient, fasted state (Figure 3A). Thus, even in a calorically replete state, stimulation of AgRP neurons alone can drive intense motivated behavior for food reinforcement.

To further investigate goal-directed behavior aimed at food acquisition, we measured physical activity (17) in AgRP-Ires-cre and wildtype mice fed ad lib and injected with either saline or CNO during the light cycle. In the first trial, food was removed simultaneously with administration of $\mathrm{CNO}$ or saline; in the second trial, food was freely available. Stimulating AgRP neurons in the absence of food led to intense, unrelenting activity, a behavioral state that continued unabated for hours (Figure 3B). We interpreted this as foodseeking behavior because the mice were often seen visiting areas of the cage where food was normally located and were also engaging in vigorous digging-type behaviors (see Supplemental Video 2). When the same mice were again given $\mathrm{CNO}$ on a different day, this time in the presence of food, this sharp increase in activity was completely absent (Figure 3B), indicating that the marked activity was directed toward the acquisition and consumption of food.

Cre-dependent AAV-DREADDs, in combination with neuronspecific cre mice, provide a technically easy means of rapidly, specifically, and reversibly regulating neuronal activity. This should empower efforts to unravel the circuitry controlling feeding and its related behaviors. Here, we found that AgRP neuron activity is both necessary and sufficient for feeding - inhibition of AgRP neurons at a time when mice normally eat (at the beginning of the dark cycle) restrains feeding, while stimulation of AgRP neurons when mice are nutritionally replete (near the beginning of the light cycle) compels feeding. Furthermore, we demonstrate that these same neurons drive not only a behavioral program to consume food, but to seek it out as well.

\section{Methods}

An expanded Methods section is provided in Supplemental Methods.

Animals. All animal care and experimental procedures were approved by the Beth Israel Deaconess Medical Center Institutional Animal Care and Use Committee. Mice from a mixed background (129/C57BL6) were housed at $22^{\circ} \mathrm{C}-24^{\circ} \mathrm{C}$ with a 12 -hour light/12-hour dark cycle with standard mouse chow and water provided ad libitum. AgRP-Ires-cre mice have been previously described (4).

Generation of $A A V-h M 3 D q-m C h e r r y$ and $A A V-h M 4 D i-m C h e r r y$ vectors. The hM3Dq and hM4Di coding sequences were cloned into a mCherry vector (18) upstream of the mCherry sequence to generate C-terminal mCherry fusion proteins. The hM3Dq-mCherry and hM4Di-mCherry coding sequences were amplified by PCR, and the amplicons and a cre-inducible $A A V$ vector with a human Synapsin 1 promoter (refs. 19 and 20; a gift from Karl Deisseroth, Howard Hughes Medical Institute, Department of Bioengineering and Department of Psychiatry and Behavioral Sciences, Stanford University, Stanford, California, USA.) were digested with NheI and AscI. The digestion products were ligated such that the coding regions for the fusion proteins were in a $3^{\prime}$ to $5^{\prime}$ orientation relative to the promoter. The final vectors were sequence verified and packaged in serotype 8 .

Stereotaxic AAV-DREADD-mCherry injections. Mice were injected as previously described (21). Briefly, 200-nL bilateral injections were made in the ARC of AgRP-Ires-cre and wild-type littermate controls (coordinates, 
bregma: anterior-posterior, $-1.40 \mathrm{~mm}$; dorsal-ventral, $-5.80 \mathrm{~mm}$; lateral, $+/-0.30 \mathrm{~mm})$. Mice were allowed 1 week to recover and then acclimated to handling for 1 week before the start of any in vivo studies.

Immunohistochemistry. Immunohistochemistry was performed as previously described (22). The primary antibodies used in these studies include rabbit anti-dsRed (Clontech; 1:2,500), chicken anti-GFP (Abcam; 1:1000), and rabbit anti-c-fos (Calbiochem; 1:25,000).

Electrophysiology. The protocols of slice preparation and whole cell recording are previously described (22). 5- to 7-week-old mice injected with AAV 10-14 days previously were used. After acquisition of stable whole cell recordings on AgRP neurons for 2-5 minutes, artificial cerebral spinal fluid solution containing 5-10 $\mu \mathrm{M}$ CNO was perfused.

Food intake studies. Food intake studies on chow were performed as previously described (4). Food intake was assessed at different time points following injection of either saline or $\mathrm{CNO}(0.3 \mathrm{mg} / \mathrm{kg})$ on alternating days in the acute studies (data shown is from male mice) or 5 days straight in the chronic studies (data shown is from female mice). Body composition (fat mass) was analyzed as previously described (4) using EchoMRI.

Energy expenditure measurements. Energy expenditure was assessed by measuring oxygen consumption as previously described (4) using a comprehensive lab animal monitoring system (CLAMS; Columbus Instruments). Data reported here are from male mice.

Nose poke assay. Operant conditioning sessions were conducted as previously described (16). Briefly, for 5 consecutive days mice fed ad libitum were injected i.p. with saline, placed in chambers, and acclimated to nose poking for food pellets for a period of 60 minutes under an FR1 operant paradigm.

Following completion of FR1 training, mice fed ad libitum were then exposed to a PR3 testing paradigm (60-minute duration) for the following 6 days and were treated with saline or $\mathrm{CNO}(0.3 \mathrm{mg} / \mathrm{kg})$ on alternate days. Data are presented as the breakpoint during the PR3. Following the completion of drug treatments, mice were fasted for 24 hours and then tested again under PR3 conditions immediately following injection with saline. Data reported herein are from male mice.

Beam-break assay. Locomotor activity was assessed as previously described (17). Briefly, individually housed mice were placed in their home cages, and ambulatory counts along the $x$ axis were recorded for 5 hours during the light cycle either in the absence or presence of food. Data reported herein are from male mice.

Statistics. Statistical analyses were performed using KaleidaGraph (Synergy Software). Overall ANOVA analyses were followed by planned pairwise comparisons between the relevant groups with a Tukey's honestly significant difference post-hoc test. A $P$ value of less than 0.05 was considered significant in these studies.

\section{Acknowledgments}

This work was supported by NIH grants (R01DK071051, R01DK075632, R01DK089044, and R37DK053477 to B.B. Lowell; R01DK069983 to E. Maratos-Flier; 1F32DK089710-01 to M.J. Krashes; U19MH82441, R01DA027120, R01MH61887, R01DA017204, RAID, and NIMH-PDSP NS064882 to B.L. Roth; and MH087074 and GM008719 to S.C. Rogan). Additionally, B.L. Roth received support from the Michael Hooker Distinguished Chair in Pharmacology, and S.C. Rogan received support from an Elion Award from the Triangle Community Foundation.

Received for publication December 24, 2010, and accepted in revised form January 26, 2011.

Address correspondence to: Bradford B. Lowell, Division of Endocrinology, Department of Medicine, Beth Israel Deaconess Medical Center, Harvard Medical School, Boston, Massachusetts 02215, USA. Phone: 617.735.3366; Fax: 617.735.3323; E-mail: blowell@ bidmc.harvard.edu.
1. Saper CB, Chou TC, Elmquist JK. The need to feed: homeostatic and hedonic control of eating. Neuron. 2002;36(2):199-211.

2. Flier JS. AgRP in energy balance: Will the real AgRP please stand up? Cell Metab. 2006;3(2):83-85.

3. Ollmann MM, et al. Antagonism of central melanocortin receptors in vitro and in vivo by agouti-related protein. Science. 1997;278(5335):135-138.

4. Tong Q, Ye C, Jones JE, Elmquist JK, Lowell BB. Synaptic release of GABA by AgRP neurons is required for normal regulation of energy balance. Nat Neurosci. 2008;11(9):998-1000.

5. Wu Q, Boyle MP, Palmiter RD. Loss of GABAergic signaling by AgRP neurons to the parabrachial nucleus leads to starvation. Cell. 2009;137(7):1225-1234.

6. Qian S, et al. Neither agouti-related protein nor neuropeptide $\mathrm{Y}$ is critically required for the regulation of energy homeostasis in mice. Mol Cell Biol. 2002;22(14):5027-5035.

7. Luquet S, Perez FA, Hnasko TS, Palmiter RD. NPY/AgRP neurons are essential for feeding in adult mice but can be ablated in neonates. Science. 2005;310(5748):683-685.

8. Gropp E, et al. Agouti-related peptide-expressing neurons are mandatory for feeding. Nat Neurosci. 2005;8(10):1289-1291.
9. Xu AW, et al. Effects of hypothalamic neurodegeneration on energy balance. PLoS Biol. 2005;3(12):e415.

10. Ferguson SM, et al. Transient neuronal inhibition reveals opposing roles of indirect and direct pathways in sensitization. Nat Neurosci. 2011; 14(1):22-24

11. Alexander GM, et al. Remote control of neuronal activity in transgenic mice expressing evolved $G$ protein-coupled receptors. Neuron. 2009;63(1):27-39.

12. Atasoy D, Aponte Y, Su HH, Sternson SM. A FLEX switch targets Channelrhodopsin-2 to multiple cell types for imaging and long-range circuit mapping. JNeurosci. 2008;28(28):7025-7030.

13 . Keesey RE, Powley TL. Body energy homeostasis. Appetite. 2008;51(3):442-445.

14. Armbruster BN, Li X, Pausch MJ, Herlitze S, Roth $\mathrm{BL}$. Evolving the lock to fit the key to create a family of $\mathrm{G}$ protein-coupled receptors potently activated by an inert ligand. Proc Natl Acad Sci U S A. 2007; 104(12):5163-5168.

15. Shin AC, Zheng H, Berthoud HR. An expanded view of energy homeostasis: neural integration of metabolic, cognitive, and emotional drives to eat. Physiol Behav. 2009;97(5):572-580.

16. Brunzell DH, Chang JR, Schneider B, Olausson P, Taylor JR, Picciotto MR. beta2-Subunit-contain- ing nicotinic acetylcholine receptors are involved in nicotine-induced increases in conditioned reinforcement but not progressive ratio responding for food in C57BL/ 6 mice. Psychopharmacology. 2006;184(3-4):328-338.

17. Pissios P, et al. Dysregulation of the mesolimbic dopamine system and reward in $\mathrm{MCH}-/-$ mice. Biol Psychiatry. 2008;64(3):184-191.

18. Shu X, Shaner NC, Yarbrough CA, Tsien RY, Remington SJ. Novel chromophores and buried charges control color in mFruits. Biochemistry. 2006;45(32):9639-9647.

19. Cardin JA, et al. Driving fast-spiking cells induces gamma rhythm and controls sensory responses. Nature. 2009;459(7247):663-667.

20. Cardin JA, et al. Targeted optogenetic stimulation and recording of neurons in vivo using cell-typespecific expression of Channelrhodopsin-2. Nat protoc. 2010;5(2):247-254.

21. Balthasar N, et al. Divergence of melanocortin pathways in the control of food intake and energy expenditure. Cell. 2005;123(3):493-505.

22. Dhillon $H$, et al. Leptin directly activates SF 1 neurons in the $\mathrm{VMH}$, and this action by leptin is required for normal body-weight homeostasis. Neuron. 2006;49(2):191-203. 